LETTER TO THE EDITOR

\title{
Optimized tokamak power exhaust with double radiative feedback in ASDEX Upgrade
}

\author{
A. Kallenbach, M. Bernert, T. Eich, J.C. Fuchs, L. Giannone, \\ A. Herrmann, J. Schweinzer, W. Treutterer, ASDEX Upgrade \\ Team \\ Max Planck Institute for Plasma Physics, EURATOM Association, Boltzmannstr. 2, \\ 85748 Garching, Germany \\ E-mail: Arne.Kallenbach@ipp.mpg.de
}

\begin{abstract}
A double radiative feedback has been developed on the ASDEX Upgrade tokamak for optimization of power exhaust with a standard vertical target divertor. The main chamber radiation is measured in real time by a subset of 3 foil bolometer channels and controlled by argon injection in the outer midplane. The target heat flux is in addition controlled by nitrogen injection in the divertor private flux region using either a thermoelectric sensor or the scaled divertor radiation obtained by a bolometer channel in the outer divertor. No negative interference of the two radiation controllers has been observed so far. The combination of main chamber and divertor radiative cooling extends the operational space of a standard divertor configuration towards high values of $\mathrm{P} / \mathrm{R}$. $\mathrm{P}_{\text {heat }} / \mathrm{R}=14 \mathrm{MW} / \mathrm{m}$ have been achieved so far with nitrogen seeding alone as well as with combined $\mathrm{N}+\mathrm{Ar}$ injection, with the time-averaged divertor peak heat flux below $5 \mathrm{MW} / \mathrm{m}^{2}$. Good plasma performance can be maintained under these conditions, namely $\mathrm{H} 98(\mathrm{y}, 2)=1$ and $\beta_{N}=3$.
\end{abstract}

PACS numbers: 52.55.Fa, 52.25.Vy, 28.52.Cx 


\section{Introduction}

ITER as well as any future tokamak or stellarator reactor demonstration device will require active control of the divertor heat flux. This will be accomplished by the injection of radiating impurities, where more than one species is required to optimize the radiative power removal in the main plasma and the divertor region [1] [2]. We foresee the combination of a low- $Z$ species for radiation in the divertor and a medium$\mathrm{Z}$ species for radiation in the outer core plasma. While in ITER the core radiative cooling will be quite limited by the requirement of a separatrix power flux being above the H-L transition power [3], in a future DEMO the core radiated power will have to be considerably higher to cope with the limited divertor radiative cooling capability. The combination and maximization of main chamber and divertor radiation is en route to the maximization of the power handling capability of a tokamak with a standard, vertical target divertor. This is often discussed in terms of the quantity $\mathrm{P} / \mathrm{R}$, the ratio of total heating power and major radius, or $\mathrm{P}_{\text {sep }} / \mathrm{R}$, using the power flow through the separatrix [4] [5]. The latter is a conservative extrapolation of the heat flux mitigation in a standard divertor, assuming that the upstream power e-folding length does not change with machine size [6]. In the simplest picture, the poloidal and radial extension of the divertor radiating layer does not change with machine size when the density is kept constant, and the total radiation increases just $\propto \mathrm{R}$ with the divertor toroidal circumference.

The radiative feedback system in ASDEX Upgrade (AUG) has been extended in order to improve its power handling capability. Real-time measurement of the main chamber radiation allows the control of the power flux into the divertor, $\mathrm{P}_{d i v}$. This can be operated simultaneously with the 'Tdiv' controller [7], which uses a measurement of the thermoelectric current in the outer divertor. The latter is a good proxy for its electron temperature but also for the peak heat flux under H-mode conditions. Since the 'Tdiv' controller uses a shunt sensor which is probably not feasible in a high heat flux divertor, a simple bolometric estimate of the divertor radiation has been implemented in addition. In combination with the main chamber radiation measurement this is used to control the target power flux, $\mathrm{P}_{\text {tar }}=\mathrm{P}_{\text {heat }}-\mathrm{P}_{\text {radmain }}-\mathrm{P}_{\text {raddiv }}=\mathrm{P}_{\text {div }}-\mathrm{P}_{\text {raddiv }}$. This paper describes the setup of the required bolometric measurements and shows examples of the combined feedback control of main chamber and divertor power, aiming towards maximisation of $\mathrm{P} / \mathrm{R}$ for a standard, vertical target divertor.

\section{Real time measurement of main chamber and divertor radiation}

ASDEX Upgrade is equipped with extensive sets of foil based bolometry. Figure 1 shows a cross section with a selection of lines of sight and a tomographic reconstruction of a high power H-mode discharge with nitrogen seeding. Since tomography is not yet feasible for real-time analysis with high temporal resolution, small sub-sets of lines of sight are used for the calculation of the radiated power and main chamber and divertor. The radiated 


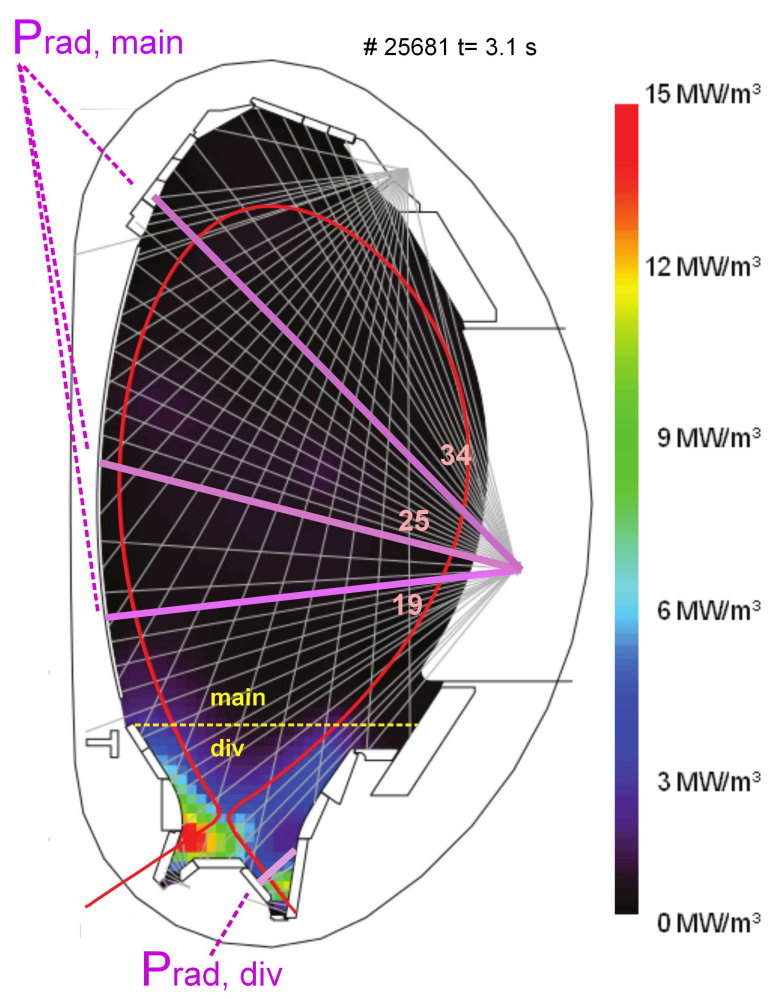

Figure 1. Cross section of AUG, radiated power densities of a high power discharge $\left(P_{\text {heat }}=14 \mathrm{MW}\right)$ and bolometer lines setup. Characteristic lines of sight are highlighted: three foil bolometer channels are used for main chamber radiation calculation and one bolometer channel is used to estimate the effect of divertor radiation on the target power load.

power is calculated by a linear combination of a few line-integrated measurements, while the weighting coefficients have been calculated by a fit to the results for a training set of 30 full tomographic reconstructions taken from 20 different seeded an non-seeded $\mathrm{H}$-mode discharges. Due to the strong gradients of the radiation in the pedestal region and inevitable uncertainties in the separatrix position, the radiated power inside the separatrix cannot be determined with good accuracy. For the use of argon as dominant main chamber radiator, evaluation of atomic data shows that the radiation comes predominantly from inside the separatrix for typical $\mathrm{H}$-mode $\mathrm{T}_{e}$ profiles [1], and $\mathrm{P}_{\text {sep }}$ is expected to be only slightly higher than $\mathrm{P}_{\text {heat }}-\mathrm{P}_{\text {radmain }}$. Tests with different sub-sets of bolometer channels showed that the main chamber radiation can be estimated with sufficient accuracy by the linear combination of 3 representative foil bolometer channels, '3f-model'. Weighting coefficients were calculated by a least-squares fit of the total main chamber radiation from tomography for the 30 test cases. Figure 2a shows a comparison of the total main chamber radiation taken from the $3 \mathrm{f}$-model with the result of the full deconvolution. Data points represent time-integrated values over typically $0.5 \mathrm{~s}$ long intervals with stationary discharge conditions. Mostly high power H-mode discharges 
are considered with different levels of nitrogen and deuterium puffing. Since the main chamber radiation is only weakly perturbed by ELMs, foil bolometry gives sufficiently accurate results. The simple $3 \mathrm{f}$-model is routinely evaluated in real time using LabView RT and transmitted to the shared memory of the discharge control system [8], where the power flux into the divertor, $\mathrm{P}_{\text {div }}=\mathrm{P}_{\text {heat }}-\mathrm{P}_{\text {radmain }}$ is calculated.

The implementation of a simple divertor radiation model as described before for the main chamber radiation turned out to be more challenging. This is due to the higher variability of divertor radiation in space and time. Different combinations of divertor viewing lines of foil bolometers were investigated for the possibility to construct a simple model for the total divertor radiation. The use of a single foil bolometer channel (1f-model) in the upper outer divertor revealed the most efficient results. The weighting factor $\mathrm{w}$ for the selected line-integrated measurement was determined by fitting to power balance, $\mathrm{P}_{\text {raddiv-1f }}=\mathrm{w}_{1 f} \mathrm{I}_{1 f}=\mathrm{P}_{\text {heat }}-\mathrm{P}_{\text {radmain-3f }}-\mathrm{P}_{\text {target }}$, where $\mathrm{I}_{1 f}$ is the line-integrated emissivity and $\mathrm{P}_{\text {target }}$ is the power load in inner+outer divertor measured by IR thermography. Finally, a good representation of both the divertor radiation from tomography as well as the power balance (fig. 2a) has been obtained. The direct fit of the 1f-model to obtain power balance shown in figure $2 \mathrm{~b}$ is essential, since the fit can partly compensate for systematic experimental errors. These include calibration uncertainties, effects of toroidal asymmetries, unaccounted ELM power losses and radiation power measured by thermography. The use of the power balance for target heat flux control is in fact not well conditioned, since the sensor value $\mathrm{P}_{\text {target,fit }}=\mathrm{P}_{\text {heat }}$ - $\mathrm{P}_{\text {radmain-3f }}-\mathrm{P}_{\text {raddiv-1f }}$ contains the difference of numbers of similar size. For the real-time application, also the heating power $\mathrm{P}_{\text {heat }}$ must be known in good accuracy by the discharge control system. Figure 2c compares the target heat flux derived from the simple radiation models with the thermography measurement. The agreement is not excellent, but considered sufficient for a feedback application.

\section{Double radiative feedback}

The 3f main chamber radiation model and the Pradddiv 1f-model have been implemented in the AUG discharge control system for simultaneous control of the power flux into the divertor and onto the targets. The main chamber radiation is controlled by argon injection through a midplane valve, while nitrogen injected through toroidally distributed valves in the divertor private flux region is used to control the divertor radiation and thus $\mathrm{P}_{\text {target }}$. Figure 3a shows time traces of a discharge where different feedback modes are used subsequently. All radiation control schemes use proportional and integral parts (PI) and cooperate smoothly, no control instabilities have been observed so far. The $\mathrm{P}_{d i v}$ controller achieves very fast reactions since the radiation build-up by Ar in the core is more effective than the divertor cooling by nitrogen, despite the more than an order of magnitude higher $\mathrm{N}_{2}$ valve flux. Instead of using divertor radiation for power balance, the Pdiv controller can also be used together with the AUG standard 'Tdiv' target heat flux controller [1]. 

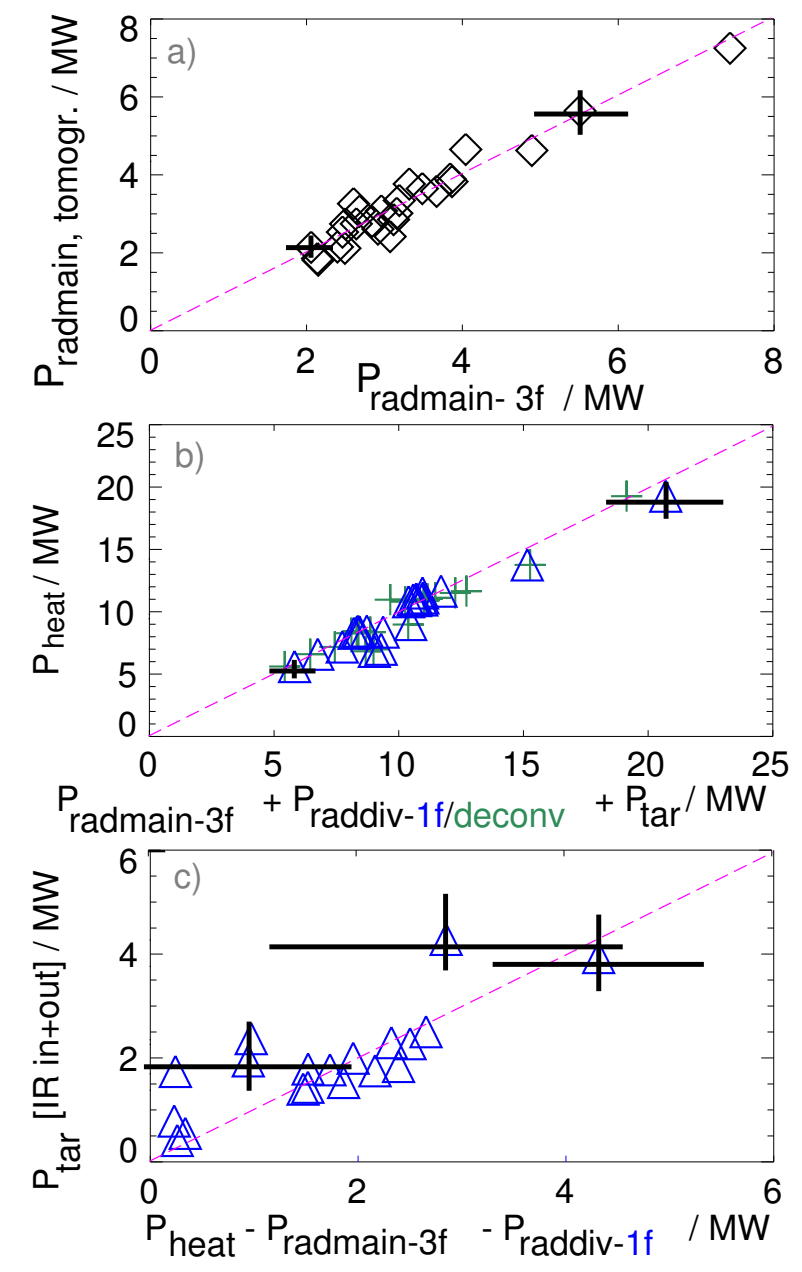

Figure 2. a) Comparison of the total main chamber radiated power from foil bolometer tomography with a simple fit model using line integrated radiation densities $\left[\mathrm{W} / \mathrm{m}^{2}\right]$ from 3 selected channels. The main chamber is defined as the region with $\mathrm{z}$ lt $-0.66 \mathrm{~m}$, so the radiation around the $X$-point is counted as divertor radiation. $b$ ) Power balance for the $1 f$ divertor radiation model and the tomographic reconstruction $(+) . c)$ Target heat flux from the simple real-time capable radiation models.

\section{High performance radiative plasmas}

Numerical predictions for the divertor behaviour in future devices like ITER and DEMO are uncertain due to the interaction of various complicated effects like anomalous transport and atomic physics processes. Numerical modelling so far fails to quantitatively reproduce detachment, in particular in the inner divertor [9]. Therefore, experimental demonstration of high values of the radiated power are required. Figure 4 shows time traces of a high $\mathrm{P} / \mathrm{R}$ discharge with $23 \mathrm{MW}$ heating power utilizing double radiative feedback with $\mathrm{N}$ and Ar. Main chamber radiation via Pdiv and divertor heat flux via 'Tdiv' are controlled simultaneously. $\mathrm{H}_{98(y, 2)}=1$ and $\beta_{N}=3$ are kept stationary, the line-averaged density $\bar{n}_{e}=10^{20} \mathrm{~m}^{-3}$ is also close to the ITER value. $\mathrm{Z}_{\text {eff }}$ is about 2 


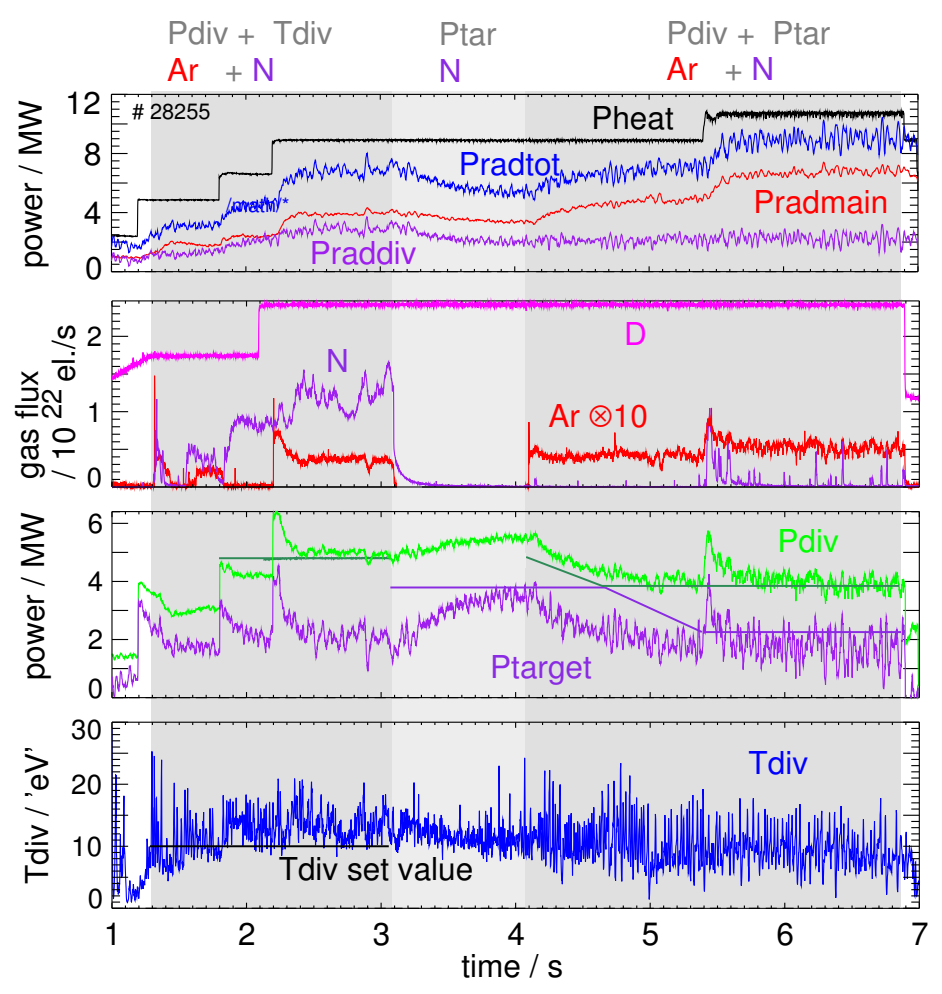

Figure 3. Time traces of a demonstration discharge with different radiative feedback modes as indicated on top, including double-feedback of power flux into the divertor, Pdiv, and divertor Tdiv as well as the target power load, Ptar, estimated by the simple bolometric calculations of main chamber and divertor radiation. The discharge is heated by up to 7.5 MW NBI, 2.2 MW ICRH and 1.8 MW ECRH. All time traces are taken from the real time protocol, with the exception of the ECRH power which was added post pulse to the Pheat, Pdiv and Ptarget signals since no reliable real-time signal was available.

during the high radiation flattop and argon is the major contributor. The good energy confinement in particular before the onset of a weak neoclassical tearing mode follows the typical hybrid like [10] and nitrogen-seeded [11] behaviour observed in AUG where good confinement is coupled to high total $\beta_{N}$ and pedestal $\beta_{N}^{p e d}$ values. The pedestal ion temperature continually rises through the heating power ramp and stays approximately constant around $\mathrm{T}_{i}^{\text {ped }}=1.3 \mathrm{keV}$ from $\mathrm{t}=2.9-4.1 \mathrm{~s}$. About $15 \mathrm{MW}$ are radiated in the main chamber, the remaining $8 \mathrm{MW}$ are well above the $\mathrm{H}$-mode threshold power of 3.4 MW according to [3], no pedestal degradation is observed. The ELM frequency is 100 $\mathrm{Hz}$ and lower compared to similar discharges with pure nitrogen seeding (with $\mathrm{f}_{E L M} \approx$ $200 \mathrm{~Hz}$ ), the tungsten concentration in the plasma is moderate at $\mathrm{c}_{W}=2 \cdot 10^{-5}$. The maximum achievable main chamber radiation with Ar seeding is expected to lie not far above the achieved value. A further reduction of the ELM frequency caused by the reduced $\mathrm{P}_{\text {sep }}$ may lead to an excessive tungsten concentration [12]. Pronounced pedestal degradation and $\mathrm{H}-\mathrm{L}$ backtransitions have been observed in pulses with too high $\mathrm{Ar}$ 

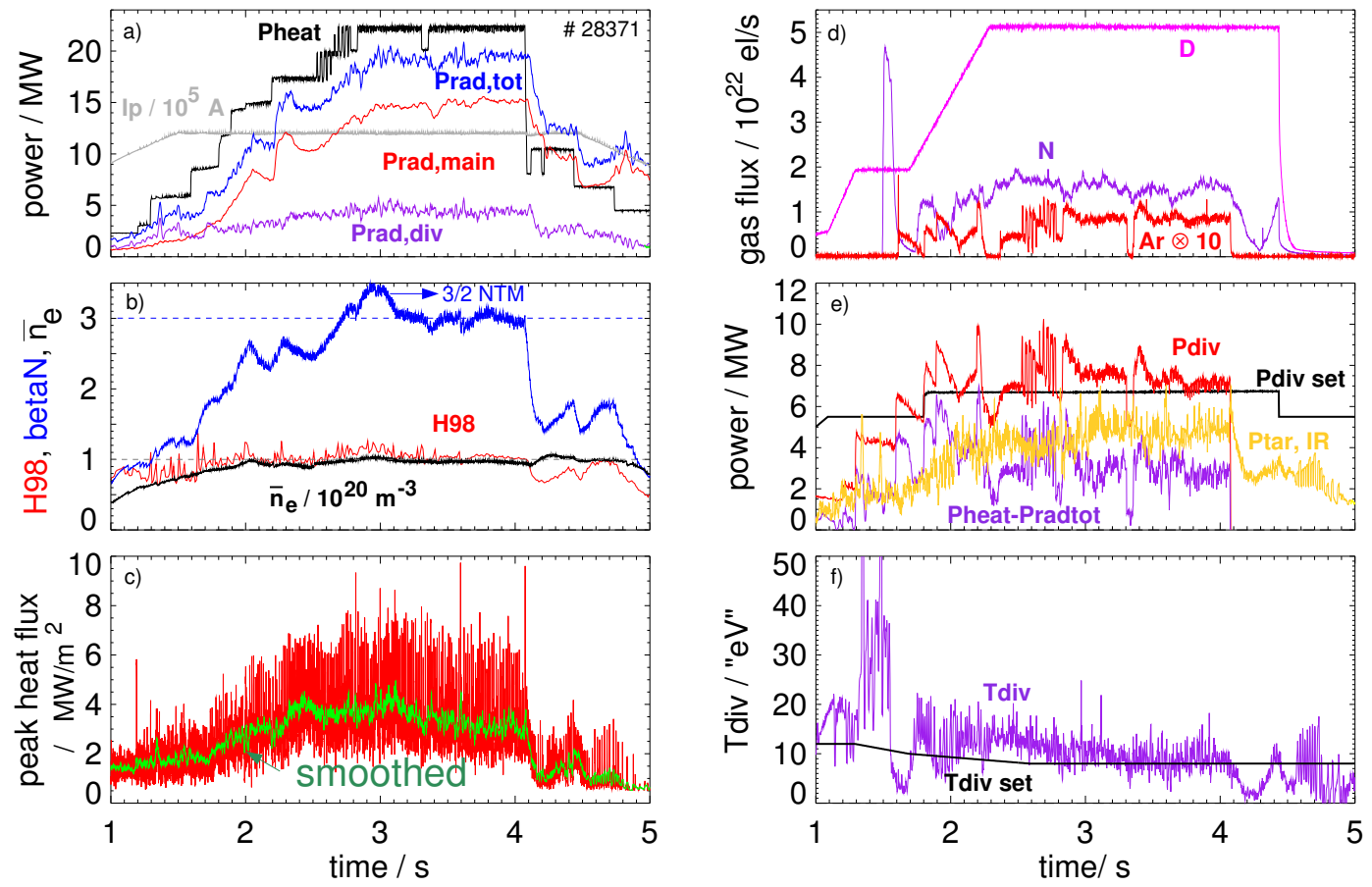

Figure 4. Time traces of a high power AUG discharge with double-feedback of power to the divertor using Ar as main chamber radiator and simultaneous target power control using nitrogen injection in the divertor private flux region. The real-time radiated power signals are corroborated by post-pulse tomography.

seeding levels when $\mathrm{P}_{\text {heat }}-\mathrm{P}_{\text {radmain }}$ approaches $\mathrm{P}_{L-H}$ according to [3]. Most of the heating power rise is dissipated by main chamber radiation, from the remaing $8 \mathrm{MW}$ during flat-top about $5 \mathrm{MW}$ are radiated in the divertor. Inspecting the $1 \mathrm{f}$-sensor for divertor radiation reveals moderate deviations of the predicted heat flux from IR measurements of the target power load by less than $2 \mathrm{MW}$ or $10 \%$ of the heating power. Langmuir probe data reveal very flat $\mathrm{T}_{e}$ profiles along the outer target decreasing from $14 \mathrm{eV}$ at $\mathrm{t}=2.5 \mathrm{~s}$ to $10 \mathrm{eV}$ at $\mathrm{t}=4 \mathrm{~s}$, the time-averaged peak heat flux and total power on the outer target vary from 5 to $4 \mathrm{MW} / \mathrm{m}^{2}$ and 2.5 to $2 \mathrm{MW}$, accordingly.

The double radiative feedback presented above allows the enhancement of the power dissipation capability in tokamaks with a standard divertor. $\mathrm{P} / \mathrm{R}=14 \mathrm{MW} / \mathrm{m}$ has been demonstrated so far in AUG. The maximum normalized power flux into the divertor while maintaining the peak heat flux below $5 \mathrm{MW} / \mathrm{m}^{2}$ is so far $\mathrm{P}_{\text {sep }} / \mathrm{R}=7 \mathrm{MW} / \mathrm{m}$ and obtained with sole $\mathrm{N}$ seeding, while the corresponding maximum main chamber radiation is $\mathrm{P}_{\text {radmain }} / \mathrm{R}=9 \mathrm{MW} / \mathrm{m}$, as shown in figure 4 . Further enhancement of $\mathrm{P} / \mathrm{R}$ in ASDEX Upgrade is expected after an upgrade of the power supply system. With the combination of high core and high divertor radiation, values beyond the ITER requirements of $\mathrm{P} / \mathrm{R} \approx 20 \mathrm{MW} / \mathrm{m}$ are assumed to be achievable with a standard divertor. A future demonstration reactor DEMO will have to rely in particular on a high main 
chamber radiation fraction to cope with the high total $\mathrm{P} / \mathrm{R}$ value, while careful control of $\mathrm{P}_{\text {sep }} / \mathrm{R}$ will be required to comply with target heat load and confinement quality limits.

\section{Acknowledgement}

Part of this work is supported by EFDA under WP12-IPH-A11-2-05. Views and opinions expressed herein do not necessarily reflect those of the European Commission.

[1] KALLENBACH, A. et al., Journal of Nuclear Materials 415 (2011) S19.

[2] NEU, R. et al., Journal of Nuclear Materials 415 (2011) S322.

[3] MARTIN, Y. R. et al., Journal of Physics: Conference Series 123 (2008) 12033.

[4] LACKNER, K., Comments Plasma Phys. Controlled Fusion 15 (1994) 359.

[5] KOTSCHENREUTHER, M. et al., Nucl. Fusion 50 (2010) 035003.

[6] EICH, T. et al., Phys. Rev. Lett. 107 (2011) 215001.

[7] KAllenBACH, A. et al., Plasma Physics and Controlled Fusion 52 (2010) 055002.

[8] GIANNONE, L. et al., Fusion Engineering and Design 85 (2010) 303.

[9] WISCHMEIER, M. et al., Journal of Nuclear Materials 415 (2011) S523.

[10] MAGGI, C. et al., Nuclear Fusion 50 (2010) 025023 (20pp).

[11] SCHWEINZER, J. et al., Nuclear Fusion 51 (2011) 113003.

[12] DUX, R. et al., Nuclear Fusion 51 (2011) 053002. 